\title{
The Security Correction of Large Consumers Long-Term Direct Power Trading Based on DC Power Transfer Distribution Factor
}

\author{
Ouyang Xu${ }^{1}$, Zhenggang Wang ${ }^{2}$, Bifeng $\mathrm{Lv}^{2}$, Jinguo Zhang ${ }^{3}$, Jiangang Yao', Bin Wang² \\ ${ }^{1}$ College of Electrical and Information Engineering, Hunan University, Changsha, China \\ ${ }^{2}$ State Grid Hunan Electric Power Company, Changsha, China \\ ${ }^{3}$ State Grid Yueyang Power Supply Company, Yueyang, China \\ Email: meetouyang@hnu.edu.cn
}

How to cite this paper: $\mathrm{Xu}, \mathrm{O}$.Y., Wang, Z.G., Lv, B.F., Zhang, J.G., Yao, J.G. and Wang, B. (2017) The Security Correction of Large Consumers Long-Term Direct Power Trading Based on DC Power Transfer Distribution Factor. Energy and Power Engineering, 9, 365-375.

https://doi.org/10.4236/epe.2017.94B042

Received: February 20, 2017

Accepted: March 30, 2017

Published: April 6, 2017

\begin{abstract}
The experiments of large consumers direct power trading is conducting in china nationwide, and it's important to the reform of electricity market. To compensated efficiencies in security correction of large consumers direct power trading, a novel security correction method based on DC power transfer distribution factor was proposed. Using the presented method to comply security correction, all the transactions that satisfy the specific requirements of maximizing social welfare are able to enter security correction process, and when the power of transmission line is out of limit, this method avoid the transaction which causes this problem is abandoned directly by introducing supplement transactions. The simulation has shown that the proposed security correction method of large consumers direct power trading based on DC power transfer distribution factor is effective.
\end{abstract}

\section{Keywords}

Large Consumers, Direct Power Trading, DC Power Transfer Distribution Factor, Security Correction, Electricity Markets

\section{Introduction}

The direct power trading between power users and power generation enterprises refers to electricity users and generation enterprises who meet the conditions for access to carry out power purchasing and selling transactions directly base on the principle of voluntary participation and independent negotiation [1]. Large consumers direct power trading is one of the direct power trading between power users and power generation enterprises, as its name implies, requires the power users have a higher voltage level and a larger electricity consumption, for 
instance, large consumers direct power trading in Hunan province requires the electricity users "receiving end voltage is above or equal to $110 \mathrm{kV}$, and last annual electricity consumption is more than 200 million.kWh".

For the purposes of electricity market change from government regulation to openness, and then establish a market-oriented mechanism, large consumers direct power trading is an inevitable part. In the market-oriented reform of electric power industry in America, Britain and Japan, large consumers direct power trading has been implemented in all these country [2] [3].

Large consumers direct power trading in China started in 2002, in the electricity system reform programme issued by The State Council, this concept was first proposed. In 2004, the National Development and Reform Commission and the State Electricity Regulatory Commission jointly issued "Interim Measures about Power Users Purchase Electricity from Generation Enterprises Directly", clarified the purposes and principles. Since 2013, especially after the release of government documents, because of its transition in the resource allocation model, introducing competition mechanisms in the sales side market and other advantages [4], large consumers direct power trading has been conducted in many provinces.

Security correction is essential to large consumers direct power trading. Out of the power system security considerations, the transaction result needs to go through security correction to determine whether it meets the constraints on power system security before it will be truly implemented. On this issue, there have been some research works at this present. The content and process of monthly security correction in provincial power company is introduced in [5]. A case study of northeast regional electricity market is presented in [6], in this paper, the problem of monthly security constrained dispatching is studied. A congestion management algorithm to minimize the cost of purchasing electricity from generation plants in generation-side electric power market is developed in [7]. Security correction system of power generation plant in east China power grid is indicated in [8]. These studies made some achievements in security correction of large consumers direct power trading, nevertheless, these research simply abandon trading outcome which doesn't meet the security constraint. Such an approach will hurt enthusiasm of consumers and power generation, is not conducive to large consumers direct power trading.

Regarding the issue above, based on DC Power Transfer Distribution Factor (PTDF), a new security correction method of large consumers direct power trading is developed in this paper. Through the introduction of supplemental transactions, this method is able to encourage large users to carry out direct electricity purchase. In this article, a mathematical model is established, and the effectiveness of the proposed method is verified by an example system.

\section{Direct Power Trading Mode}

\subsection{Transaction Model}

At present, there are primarily two types of large consumers direct power trad- 
ing modes, namely direct negotiation transaction and centralized matching transaction. Direct negotiation transaction means consumers and generator companies conclude the transaction through bilateral and independent negotiation, and transaction result will be submitted to the Independent System Operator (ISO). Direct negotiation transaction is flexible and easy to implement, so it's the main way to carry out large consumers direct power trading at an initial stage. However, it also has characteristics such as small competition intensity and opaque price signal [9].

In the background of the market-oriented reform of generation-side electric power market has been carried out for many years and the power market technical support system has been perfected, compared with direct negotiation transaction, centralized matching transaction is more suitable for large consumers direct power trading, and is beneficial to the construction of the market in the future [2].

To maximize social welfare, centralized matching transaction obtains transaction result according to generators and large users of their declared transfer quantity and price. The exact process is as follows. First of all, sort declared price of buyer from high to low and declared price from seller low to high, then match these price one by one, the difference between buyer's price and seller's price is greater than or equal to zero is effective transaction. Effective transaction determines transaction volume, if there is surplus, then enter the top of the queue to continue until the transaction is complete. The mathematical model is:

$$
F=\max \sum_{i \in U} \sum_{j \in G}\left[\left(p_{i}-p_{j}\right) \cdot Q_{i j}\right]
$$

where $F$ is social welfare, $U$ is set of large users, $G$ is set of power generation enterprises, $p_{i}$ and $p_{j}$ is declared price of buyer and seller respectively, $Q_{i j}$ is transaction volume which is given by:

$$
Q_{i j}=\min \left(Q_{i}, Q_{j}\right)
$$

where $Q_{i}$ is declared transfer quantity of large users $i, Q_{j}$ is declared transfer quantity of large users $j$.

The constraints of the model are as follows:

1) Power quantity balance

$$
\sum_{i \in U} Q_{i}-\sum_{j \in G} Q_{j}=0
$$

2) Active power flow constraint of transmission line

$$
\left|\mathrm{P}_{l}\right| \leq \mathrm{P}_{l}^{\max }
$$

3) Total transaction amount

$$
\sum Q_{i j} \leq Q_{\max }
$$

where $\mathrm{P}_{l}$ is active power of transmission line $l, \mathrm{P}_{l}^{\max }$ is maximum active power flow of transmission line $l, Q_{\max }$ is maximum amount of large consumers direct power trading determined by ISO. 


\subsection{Security Correction}

In the actual trading, based on the above-mentioned centralized matching transaction model, security correction process is outlined in the following. First, for the model that does not consider the active power flow constraint of transmission line, solve the initial transaction result by the conventional optimization method or the fast algorithm [10]. And then, for each specific transaction in the initial transaction result, execute security correction in accordance with the price differential spread from high to low. Finally, determine whether the transmission line security constraints are fulfilled. When a specific transaction leads to the line out of its constraints, then give up this transaction directly. Repeat this process until all transactions have been checked.

This method is easy and fast, which embodies the principle of marketization. However, there are some problems with this method. For example, the impact of different transactions on the same line may be opposite. So, when a specific transaction executes security correction priority, it may fail and be discarded, but when the subsequent transaction joining, this specific transaction can pass the correction somehow [11]. The existence of this situation will result in the abandonment of some transaction which could have been carried out. Moreover, there are also disadvantages to set constraint of total transaction amount in Equation (5). Although it can guarantee the security and stability of the electricity market, but also may lead to some transaction which could have been to maximize social welfare cannot be implemented. The above-mentioned problems, in a manner, is a blow for large consumers direct power trading.

\section{DC Power Transfer Distribution Factor}

\subsection{Power Flow}

For the branches with node $i$ and node $j$ at both ends, the active power flow equation can be written as:

$$
P_{i j}=\left(V_{i}^{2}-V_{i} V_{j} \cos \theta_{i j}\right) g_{i j}-V_{i} V_{j} \sin \theta_{i j} b_{i j}
$$

where $P_{i j}$ is power flow of branch, $V_{i}$ and $V_{j}$ is voltage of node $i$ and node $j, \theta_{i j}$ is angular phase difference of branch, $g_{i j}$ is branch conductance, $b_{i j}$ is branch susceptance [12].

For normal transmission network, the node voltage is equal to rated voltage, angular phase difference of branch is small, and the line resistance is much smaller than the reactance, that is, $V_{i}=V_{j}=1, \theta_{i j} \simeq 0, r_{i j}=0$, so Equation (6) can be simplified into:

$$
P_{i j}=-b_{i j}^{\prime}\left(\theta_{i}-\theta_{j}\right)=\frac{\theta_{i}-\theta_{j}}{x_{i j}}
$$

where $b_{i j}^{\prime}=-1 / x_{i j}, x_{i j}$ is branch reactance. Because power of node $i$ is balance, consequently,

$$
P_{i}=\sum_{j \in i, j \neq i} P_{i j}=\sum_{j \in i, j \neq i} \frac{\theta_{i}-\theta_{j}}{x_{i j}} \quad i=1,2, \cdots, n
$$


where $P_{i}$ is injected active power of node $i$. For each node in the network except balance node, write the above equation, the matrix expression of DC power flow can be obtained:

$$
\boldsymbol{P}=\boldsymbol{B} \boldsymbol{\theta}
$$

where $\boldsymbol{B}$ is $n \times n$ order node admittance matrix, matrix element is:

$$
\left\{\begin{array}{l}
B_{0}(i, i)=\sum_{j \in i, j \neq i} \frac{1}{x_{i j}} \\
B_{0}(i, j)=-\frac{1}{x_{i j}}
\end{array}\right.
$$

\subsection{Transfer Distribution Factor}

When active power of node $i$ change $\Delta P_{i}$, from Equation (9), the corresponding variation of node voltage phase angle is:

$$
\Delta \theta=X\left(e_{i} \Delta P_{i}\right)=X_{i} \Delta P_{i}
$$

where $e_{i}$ is unit column vector, the value in the corresponding position of node $i$ is $1, X$ is inverse matrix of node admittance matrix $\boldsymbol{B}, X_{i}$ is the $i$-th column vector of $X$.

Assume $M_{k}$ is node-branch incident matrix of branch $k$, and inflow is +1 , outflow is -1 , when active power of node $i$ change, the homologous variation of active power flow of branch $k$ is:

$$
\Delta P_{k}^{i}=\frac{M_{k}^{T} \Delta \theta}{x_{k}}=\frac{1}{x_{k}} M_{k}^{T} X_{i} \Delta P_{i}=G_{k-i} \Delta P_{i}
$$

where $G_{k-i}=\frac{1}{x_{k}} M_{k}^{T} X_{i}$ is transfer distribution factor between branch $k$ and node $i$.

Specific to large consumers direct power trading, let the nodes of branch $k$ is node $m$ and node $n$, when power generation enterprises (node $i$ ) and large consumers (node $j$ ) make a transaction with amount of $\Delta P_{i j}$, sincetransaction between power generation enterprises and large consumers subject to Equation (3), the active power of node $i$ and node $j$ change $\Delta P_{i j}$ and $\Delta P_{i j}$ respectively, substitute this into Equation (12), transfer distribution factor in this situation is:

$$
\begin{aligned}
G_{k-i j} & =\frac{1}{x_{k}} M_{k}^{T} X\left[e_{i} \vdots e_{j}\right]\left[\begin{array}{c}
1 \\
-1
\end{array}\right] \\
& =\frac{x_{m i}-x_{m j}-x_{n i}+x_{n j}}{x_{k}}
\end{aligned}
$$

From Equation (13) we conclude that PTDF is only correlative with topology of transmission network, hence it's easy to calculate and use.

\section{Security Correction Based on DC PTDF}

Suppose $C=\left(c_{1}, \cdots, c_{i}, \cdots, c_{n}\right)$ is a set of large consumers direct power trading preliminary result which haven't executed security correction yet, and $c_{i}$ is one 
specifically transaction one of the result, subscript $i$ indicates the price differential spread from high to low, so the security correction process described in 2.2 is shown in Figure 1.

To solve the problems of security correction in this process, calculate DC PTDF $\delta_{i-k}$ between branch $k$ and transaction $c_{i}$ by using Equation (13), and modify security correction process as shown in Figure 2, where $M$ is mark vector, in the security correction process, transaction resulting in transmission line out of its constrain and number of line will be remarked.

After all the specific transaction have been checked, execute security correction again to determine whether there is transaction which is unable to meet the constraint of security, if there is no transmission line out of power limitation, then the process is end, otherwise, supplemental transaction is performed.

Let $C^{\prime}=\left(C_{1}^{\prime}: C_{2}^{\prime}\right)=\left(c_{1}^{\prime}, \cdots, c_{j}^{\prime}: c_{j+1}^{\prime} \cdots, c_{m}^{\prime}\right)$ is set of available supplemental transaction, $\delta_{j-k}$ is DC PTDF between branch $k$ and transaction $c_{j}^{\prime}$, hence supplemental transaction should satisfy Equation (14):

$$
\left\{\begin{array}{l}
\delta_{i-k} \cdot \delta_{j-k}<0 \\
\frac{\mathrm{P}_{i}^{a f t e r}}{\mathrm{P}_{i}^{\max }}<\xi
\end{array}\right.
$$

where $\mathrm{P}_{i}^{\text {after }}$ is active power of normal transmission line, the adjusted power is taken into account, it can be calculated from the original transmission power, supplemental transaction value and Equation (13), $\mathrm{P}_{i}^{\max }$ is the maximum transmission power of this line, $\xi$ is power margin percentage.

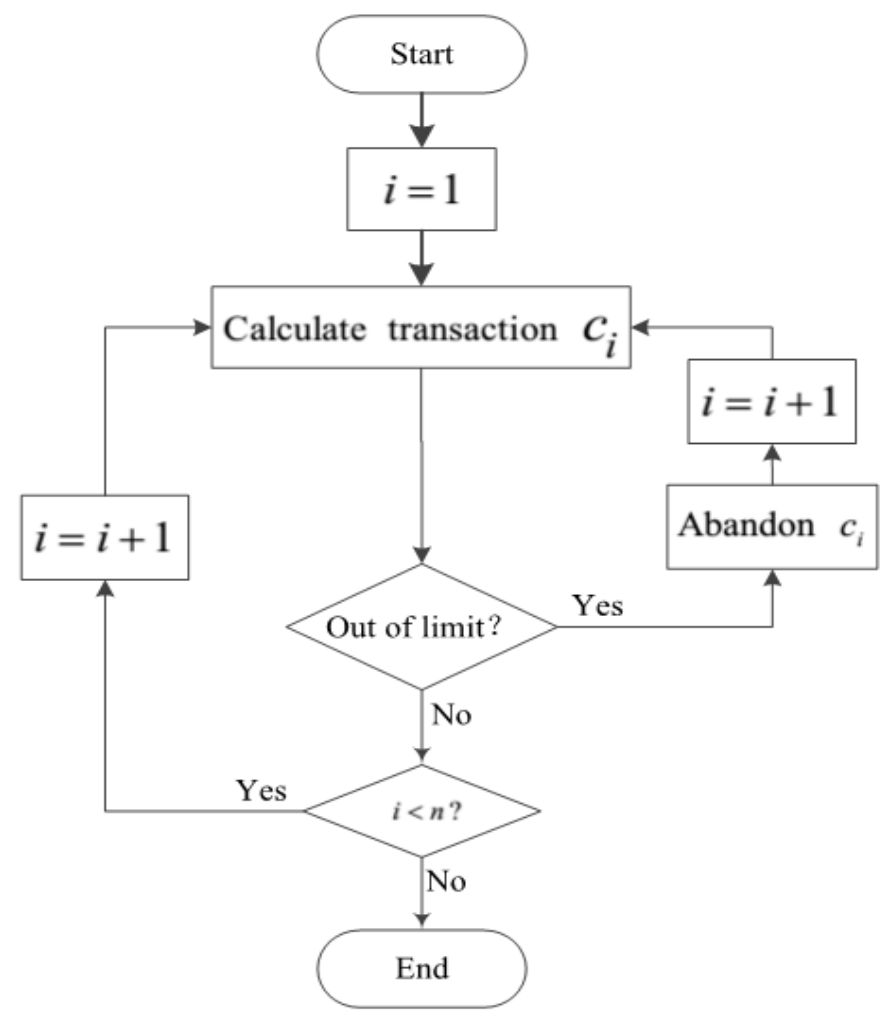

Figure 1. Flow chart of original security correction. 


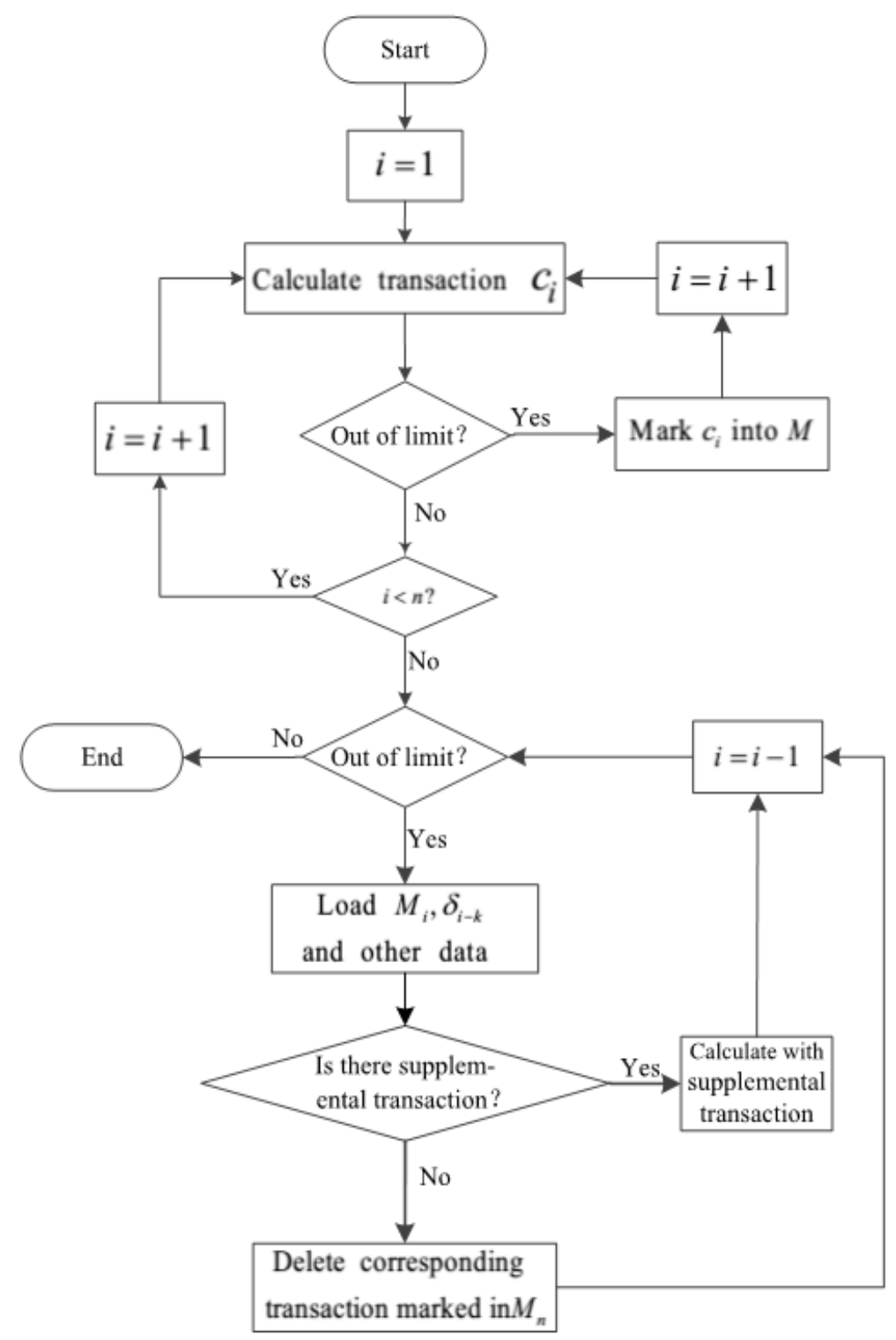

Figure 2. Flow chart of improved security correction.

The above conditions reflect two principles of choosing a supplemental transaction. First, it is able to reduce the load of the transmission line which is out of power limitation. Secondly, it will not exacerbate load on other lines. If there is no supplemental transaction to meet the conditions, according to the mark vector $M$, forward exclude transaction which will lead to the line exceed its limitation, repeat the above procedure until security correction is ended.

The exact composition of the supplemental transaction set is as follows. $C_{1}^{\prime}$ is set of transaction whose social welfare is greater than 0 but is unable to carry out because of total transaction amount constraint, $C_{2}^{\prime}$ is set of transactions whose social welfare is less than 0 . For set $C_{1}^{\prime}$, these transactions cannot be implemented in original security correction due to total transaction amount constraint. However, in the new security correction, there may be a transaction that is beneficial to the security of the system, so it's reasonable to provide a separate 
trading amount for such transaction as a "reward". For set $C_{2}^{\prime}$, these transactions are unable to meet the requirement of maximum social welfare, so it's impossible for them to win the bid in the original security correction. Nevertheless, in the electricity market, the successful bidder may negotiate with power generation and users in set $C_{2}^{\prime}$ in order to successfully pass security correction, and make the transaction of set $C_{2}^{\prime}$ into a transaction of set $C_{1}^{\prime}$. Set $C_{2}^{\prime}$ is designed separately is to ensure that improved security correction in the more open market is still valid.

\section{Simulation}

In order to verify the feasibility and effect of the method, a verification simulation is implemented in the IEEE New England test system, the system wiring diagram is shown in Figure 3.

There are three assumptions in the simulation calculation:

1) Large consumers direct power trading is mainly quarterly and monthly transactions, in this simulation, choose the monthly transaction as the type and electricity of every transaction distribute to one month (30 days) equally.

2) To simplify the calculation, only the load of large consumers direct power trading is taken into account, other load is not included.

3) Supplemental transaction is formed by set $C_{1}^{\prime}$ only.

Let the total transaction amount is $1600 \mathrm{GW} \cdot \mathrm{h}, \xi$ is 0.9 , take branch TL1314 between node 13 and node 14 as the analysis object, its active power limitation is $60 \mathrm{MW}$, power flow from node 14 to node 13 is positive. Examine the variety of active power of TL1314 in the simulation process to verify the effectiveness of the new method.

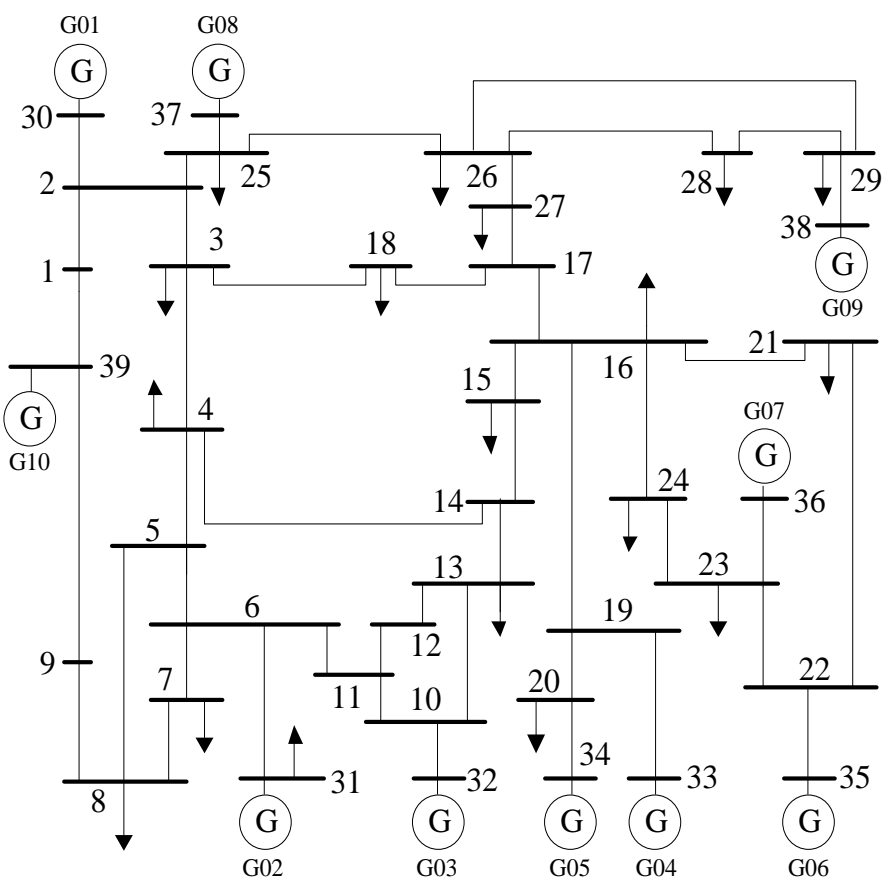

Figure 3. IEEE New England test system. 
Specific information of the transactions is shown in Table 1. Social welfare of each transaction in the table is greater than 0 and permutation of each transaction spread from high to low based on the price differential between declared buyer's price and seller's price. So we can easily conclude that No.5 and No.6 transaction are unable to enter security correction process due to total transaction amount constraint.

On the basis of these data, execute security correction process for each transaction, the active power variation of TL1314 is illustrated as below.

Figure 4 shows that the first three transactions will not lead to TL1314 active power out of limit, but when the fourth transaction is taken into consideration, power of TL1314 is out of its limitation. Hence the No. 4 transaction will be discarded directly in original security correction.

Now calculate DC PTDF between TL1314 and transaction which leads to power out of limit (i.e. No. 4 transaction), and calculate DC PTDF between TL1314 and transaction in the supplemental transaction set (i.e. No. 5 and No. 6 transaction). The results are in Table 2.

According to Equation (14), No. 5 transaction is chosen as supplemental transaction, and active power of TL1314 decline to $40.63 \mathrm{MW}$, this implies that security correction result is satisfactory. In this situation, No. 1 to No. 4 transactions is normal transactions, No. 5 transaction is supplemental transaction, compared with the original method, No. 4 transaction is not abandoned and solves the problem that No. 5 transaction is unable to enter security correction process due to total transaction amount constraint. In summary, new method is more flexible and effective.

\section{Conclusions}

This paper proposes a new security correction method which is intended to solve the problem that the total transaction quantity of large consumers direct power trading is limited and the transaction result may be directly discarded during

Table 1. Information of direct trading.

\begin{tabular}{cccc}
\hline $\begin{array}{c}\text { Transaction } \\
\text { No. }\end{array}$ & Generator No. & User Node No. & $\begin{array}{c}\text { Trading volume } \\
(\mathrm{GW} \cdot \mathrm{h})\end{array}$ \\
\hline 1 & G08 & 13 & 500 \\
2 & G09 & 15 & 550 \\
3 & G01 & 8 & 500 \\
4 & G07 & 7 & 400 \\
5 & G03 & 4 & 450 \\
6 & G06 & 3 & 500 \\
\hline
\end{tabular}

Table 2. The value of DC power transfer distribution factor.

\begin{tabular}{cccc}
\hline Transaction No. & 4 & 5 & 6 \\
\hline DC PTDF & -0.3571 & 0.51127 & -0.08064 \\
\hline
\end{tabular}




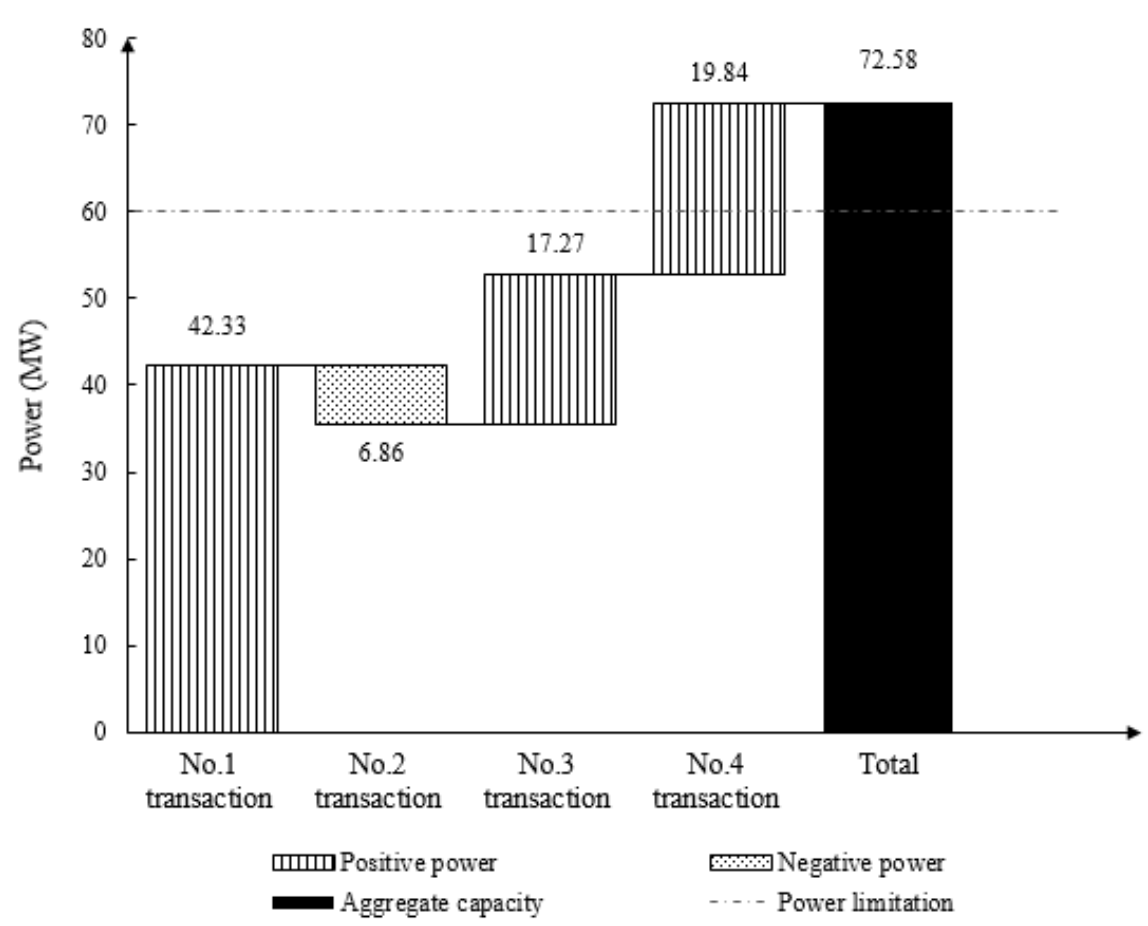

Figure 4. Active power variation of TL1314.

security correction. The new approach based on DC power transfer distribution factor that is only correlative with the network structure and parameter. Moreover, supplemental transactions are introduced to the transactions that do not meet the requirement of security correction, so that all the transactions which can maximum social welfare is able to enter security correction progress and avoid being discarded directly.

The validity of the proposed method is demonstrated by a simulation. The result implies that in the case of the introduction of supplemental transactions, large consumer direct purchase transactions that will be discarded in the existing security correction process have been retained. This is significant for encouraging large users and power plants participate in transactions actively and beneficial to promotion of large consumers direct power trading.

\section{References}

[1] Wang, P., Wu, S.H., Dai, J.L., et al. (2009) Research on Model and Mechanism of Large Consumers Direct-purchasing Based on Incentive Compatibility Principle. Proceedings of the CSEE, 10, 91-96.

[2] Zhang, X., Liu F.B., Peng T., et al. (2014) A Discussion on Key Issues for Direct Trading Between Power Users and Plant. Automation of Electric Power Systems, 13, 33-37. http://dx.doi.org/10.7500/AEPS20131014014

[3] Liu, F.B., Peng, T., Zhang, X., Pang, B. and Han, Y.(2013) Pilot and Promotion Recommendations for Direct Trading between Power Users and Plants. East China Electric Power, 9, 1941-1944.

[4] Xia, Q., Bai, Y., Zhong, H.W. and Chen, Q.X. (2013) Institutional Design and Suggestions for Promotion of Direct Electricity Purchas by Large Consumers in China. 
Automation of Electric Power Systems, 20, 1-7. http://dx.doi.org/10.7500/AEPS20130913013

[5] Li, J., Liu, D.N., Chen, Z.Y., Wang, H.C. and Zeng, M.(2011) Research of Security Constrained Dispatching for Monthly Generation Plan. Proceedings of 2011 AASRI Conference on Applied Information Technology, 95-98.

[6] Wang, G.T., Ding, P., Liu, X., et al. (2004) Sectional Security Constrained Dispatching in Northeast Regional Power Market. Power System Technology,10, 18-21+33. http://dx.doi.org/10.13335/j.1000-3673.pst.2004.10.005

[7] Wu Y.G., Deng Y.M., Zheng R., Lei J.S. andJin, Z.H.(2001) A Security Correction Algorithm for the Generation Market. Proceedings of the CSEE, 6, 49-53. http://dx.doi.org/10.13334/j.0258-8013.pcsee.2001.06.012

[8] Ge, C.J., Wang, D.X., Ge, M.H., et al. (2008) Security Checking System for Daily Generation Scheduling of East China Power Grid and Its Expansion. Automation of Electric Power Systems, 10, 45-48.

[9] Chen, H.Y., Zhang, S.L. and Zhang, Y.(2008) Research on Transaction Mode of Direct Power Purchase by Large Consumers in Electricity Market. Power System Technology, 21, 85-90. http://dx.doi.org/10.13335/j.1000-3673.pst.2008.21.020

[10] Chen, X.Y. and Hu, J.Y.(2008) Preliminary Discussion on the Straight Power Transactions Between LargePower Users and Generation Enterprises. Automation of Electric Power Systems, 24, 100-103. http://dx.doi.org/10.3321/j.issn:1000-1026.2008.24.023

[11] Xiao, J. and Wen, F.S. (2008) Congestion Dispatch for Generation Rights Trade. Automation of Electric Power Systems, 18, 24-29. http://dx.doi.org/10.3321/j.issn:1000-1026.2008.18.005

[12] Zhang, B.M., Chen, S.S. and Yan, Z. (2007) Advanced Electric Power Network Analysis. 2nd Edition.Tsinghua University Press, Beijing.

Submit or recommend next manuscript to SCIRP and we will provide best service for you:

Accepting pre-submission inquiries through Email, Facebook, LinkedIn, Twitter, etc. A wide selection of journals (inclusive of 9 subjects, more than 200 journals)

Providing 24-hour high-quality service

User-friendly online submission system

Fair and swift peer-review system

Efficient typesetting and proofreading procedure

Display of the result of downloads and visits, as well as the number of cited articles

Maximum dissemination of your research work

Submit your manuscript at: http://papersubmission.scirp.org/

Or contact epe@scirp.org 